Original Paper http://ajol.info/index.php/ijbcs http://indexmedicus.afro.who.int

\title{
Effet des amendements organiques sur la croissance et le rendement de la pomme de terre (Solanum tuberosum) cultivée sur un sol dégradé dans la région de Kabinda, République Démocratique du Congo
}

\author{
Adolphe Nsomue NGOYI ${ }^{*}$, Gustave Kishiko MASANGA ${ }^{2}$, \\ Hubert Mulungu BILA ${ }^{1}$, Alphonse Yangoy YASHIMA ${ }^{3}$, \\ Médard Matala MILAMBO ${ }^{4}$, Laurent Nsapu NDJIBU ${ }^{5}$ et Louis Longanza BABOY ${ }^{6}$ \\ ${ }^{1}$ Département de Phytotechnie, Université Notre Dame de Lomami, Province de Lomami, République \\ Démocratique du Congo. \\ ${ }^{2}$ Département de Zootechnie, Université Notre Dame de Lomami, Province de Lomami, République \\ Démocratique du Congo. \\ ${ }^{3}$ Département d'Economie agricole, Université Notre Dame de Lomami, Province de Lomami, République \\ Démocratique du Congo. \\ ${ }^{4}$ Département de Développement Rural, Institut Supérieur d'Etudes Agronomiques et Vétérinaires de Lukashiyi \\ (ISEAV/LUKASHIYI), Province de Lomami, République Démocratique du Congo. \\ ${ }^{5}$ Province de Lomami, République Démocratique du Congo. \\ ${ }^{6}$ Département de Phytotechnie, Université de Lubumbashi, Province du Haut-Katanga, République \\ Démocratique du Congo. \\ *Auteur correspondant ; E-mail : ngoyiadolphe8@gmail.com, Tél : (243) 996984156
}

\section{RESUME}

Le bas niveau de la fertilité des sols à Kabinda et la rareté des engrais chimiques sur le marché orientent les agriculteurs à recourir à une fertilisation palliative et durable. Cette étude a été réalisée dans le but d'évaluer l'effet des amendements organiques sur la croissance et le rendement de la pomme de terre. L'essai était installé suivant un dispositif en blocs complets randomisés. Les traitements en cinq répétitions ont été constitués d'un témoin (T0), de la bouse de vache (T1), de cendres (T2) et de la biomasse fraiche de Entada abyssinica (T3). Les observations ont porté sur les paramètres de croissance et de rendement et l'analyse de la variance (ANOVA) a été appliquée pour la comparaison. Les résultats obtenus renseignent que la pomme de terre se comporte positivement sous effet d'amendements $(\mathrm{P}<0.05)$ comparativement au sol non amendé. Le plus grand nombre de feuilles est démarqué nettement au traitement à base des cendres avec 4 feuilles contre 2 feuilles pour le témoin. L'apport de la cendre à la dose de $10 \mathrm{~kg} / \mathrm{ha}$ sur la culture de la pomme de terre a augmenté le rendement de 1 t/ha par rapport au sol non amendé. L'utilisation de ces amendements dans une région comme Kabinda contribuerait au développement de l'agriculture en luttant contre l'insécurité alimentaire et la pauvreté. Ainsi donc, L'utilisation de la cendre est recommandable pour la production de la pomme de terre vu ses effets sur la culture et sa disponibilité dans la ville de Kabinda.

(C) 2020 International Formulae Group. All rights reserved.

Mots clés : Fertilité, engrais chimiques, production, Solanum tuberosum, Kabinda. 


\title{
Effect of organic amendments on growth and yield of potato (Solanum tuberosum L.) on soil degraded in Kabinda area, Democratic Republic of Congo
}

\begin{abstract}
The low level of soil fertility in Kabinda and the scarcity of chemical fetilizers on the market guide farmers to seel palliative and sustainable fertilization. This study was carried out in order to assess the effect of organic amendments on the growth and potato yield. The trial was installed using a ramdomized complete block system. Five Treatments in five repetitions, consisted of a witness (T0), cow dung (T1), ash (T2) and Entada abyssinica fresh biomass (T3). Observations focused on growth and yield parameters and analysis of variance (ANOVA) at the 5\% threshold was applied for comparison betwen treatments. The results obtained indicate that the potato behaves positively under the effect of amendments $(\mathrm{P}<0.05)$ compared to unamended soil. The greatest leaves number is clearly demarcated in the ash based treatment with 4 leaves versus 2 leaves for the witness treatment. The addition of ash to the potato crop increased the yield by $1 \mathrm{t} / \mathrm{ha}$ compared to the unamended soil. The use of ash is recommendable for the production of potato in the city of Kabinda.
\end{abstract}

(C) 2020 International Formulae Group. All rights reserved.

Keywords : Fertility, chemical fertilizers, production, Solanum tuberosum, Kabinda

\section{INTRODUCTION}

Selon les statistiques de la FAO (2012), environ 870 millions de personnes seraient sous-alimentées, soit $12,5 \%$ de la population dans le monde. L'agriculture africaine est confrontée à un défi de taille dans la mesure où elle a besoin de doubler la production alimentaire d'ici à 2030 (FAO, 2016). Honfoga (2007) démontre que l'augmentation de la production agricole en Afrique Subsaharienne a connu une chute dans les années 1960 et 1980 $(2,6 \%$ à $0,9 \%)$ et un relai dans les années 1990 $(2,8 \%)$; toutefois, cette production ne suffit pas pour répondre aux besoins dus à un surpeuplement. La pomme de terre (Solanum tuberosum) occupe une place importante dans l'alimentation de base des populations dans les pays en développement. La culture de la pomme de terre offre de nombreux atouts: d'un point de vue agronomique, sa culture est aisée, son potentiel de rendement est important (20 à $30 \mathrm{t} / \mathrm{ha}$ ). Du point de vue nutritionnel, elle se classe parmi les plantes à tubercule les plus nutritives avec une teneur énergétique élevée. $\mathrm{Du}$ point de vue commercial, elle est très appréciée par les populations congolaises. Certaines études démontrent qu'en Algérie par exemple, la consommation de la pomme de terre est de $60 \mathrm{~kg}$ par personne et par an, et elle constitue une culture de rente pour de nombreux agriculteurs.

Dans la région de Kabinda, la pomme de terre est quasiment absente dans les ménages agricoles et sur le marché. Parmi les causes qui sont à la base de cette rareté de la pomme de terre à Kabinda, nous illustrons l'absence criarde de la politique agricole au niveau de l'Etat, le manque de vulgarisation de la culture, l'ignorance des techniques culturales de la pomme de terre, mais surtout le manque d'un bon matériel de semis pour la plantation (Mukendi et al., 2017b).

Néanmoins pour la plupart de problème accusés à la culture de la pomme de terre dans la ville de Kabinda, certains peuvent avoir de solutions sur le plan agronomique qui sont même socioculturelles, s'il y a la volonté de faire la réalisation de cette culture dans le milieu enfin de pallier au problème de l'insécurité alimentaire (IFDC, 2002 ). Dans le même ordre d'idée, l'intérêt porté sur la culture de la pomme de terre est manifeste tant en Amérique (Colombie, Brésil) qu'en Afrique (Cameroun, Côte-d'Ivoire, Bénin, Togo, Nigéria). Cependant, cette culture ne donne pas toujours des rendements attendus, nécessitant ainsi une fertilisation afin de maintenir ou 
d'améliorer la fertilité des sols par l'apport des éléments nutritifs (Ognalaga et al., 2017). La bouse de vache et la biomasse de Entada abyssinica ont des capacités d'améliorer les propriétés physico-chimiques et biologiques du sol en produisant de l'humus (Orwa et al., 2009 ; Biaou et al., 2017 ; Yalombe et al., 2017). L'apport de la cendre au sol dégradé permet de corriger la structure et l'acidité du sol en fournissant des éléments nutritifs tels que les cations calcium $\left(\mathrm{Ca}^{++}\right)$et le magnésium $\left(\mathrm{Mg}^{++}\right)$aux plantes. Les cultures installées sur le sol enrichi à la matière organique à base de la cendre donnent des rendements supérieurs comparativement aux autres matières organiquestable (Lickaz, 2002). La cendre permet également de maintenir ou d'élever le $\mathrm{pH}$ du sol jusqu'aux gammes optimales voulues et la fixation réversible du phosphore sur les complexes argilo-humique (Yalombe et al., 2017). La connaissance de ces amendements permettra de mettre à la disposition des maraîchers des types matières organique en vue d'une production durable de la pomme de terre sur les sols sableux.

L'objectif de notre étude est d'évaluer les effets de la bouse de vache, de la cendre et de la biomasse de Entada abyssinica sur la croissance et le rendement de la pomme de terre dans les conditions agroécologiques de Kabinda. L'hypothèse émise est l'une des matières organiques apportées au sol influencerait positivement la croissance et/ou le rendement de la pomme de terre.

\section{MATERIEL ET METHODES Milieu d'étude}

L'étude a été menée au champ expérimental de la Faculté des Sciences Agronomiques de l'Université Notre Dame de la Lomami, dans la province de Lomami en République Démocratique du Congo. Les coordonnées géographiques du site expérimentales sont $24^{\circ} 33^{\prime} \mathrm{E}, 6^{\circ} 06^{\prime} \mathrm{S}$ et 840 m d'altitude (Mukendi et al., 2017b). Le climat caractéristique du site expérimental est de type Aw3 selon la classification de Köoppen. C'est un climat tropical humide caractérisé par l'alternance de 2 saisons : une pluvieuse qui dure 9 mois (d'août à avril) et une saison sèche de 3 mois (de mai à juillet). La pluviosité moyenne annuelle atteint $1600 \mathrm{~mm}$ et la température annuelle moyenne oscille autour de $25{ }^{\circ} \mathrm{C}$. La quasi-totalité du sol sur le site a un caractère acide, généralement appartenant au groupe de ferralsols d'après la classification de l'INEAC de 1961 (Ministère du plan, 2005). Ce sol léger, facilement drainé et lessivé, avec une capacité de rétention d'eau très faible (sols pauvres à faible valeur agricole). La végétation est dominée par les espèces graminéennes, notamment: Hyperhenia spp, Andropogon spp, Abyssinica et Imperata cylindrica.

\section{Méthodes}

L'essai a été conduit pendant la première saison de pluie 2017-2018. La préparation du lit de semence s'est faite manuellement avec la houe dont la profondeur de labour est estimée à $15 \mathrm{~cm}$. Pour recueillir les données statistiquement comparables, l'expérimentation a été conduite suivant un dispositif en blocs complets randomisés avec cinq répétitions. Les traitements ayant fait l'objet de cette étude étaient composés d'un témoin sans amendement (T0), de la bouse de vache (T1), de cendres (T2) et de la biomasse de E. abyssinica (T3). La superficie d'une parcelle était de $5 \mathrm{~m}$ de longueur et $1,30 \mathrm{~m}$ de largueur et la superficie totale de l'essai était de 3,9 ares. La distance entre les espaces élémentaires était $50 \mathrm{~cm}$. Une semaine après la préparation du lit de semence, nous avons procédé à l'enfouissement de la bouse de vache, de la biomasse de Entada abyssinica et de la cendre comme engrais organique. Ces engrais organiques étaient enfouis d'une manière localisée dans les trous de plantation à une profondeur de $15 \mathrm{~cm}$. Les quantités apportées pour chacune des matières organiques a été de $60 \mathrm{~kg} / \mathrm{ha}$ de bouse de vaches, $80 \mathrm{~kg} / \mathrm{ha}$ de biomasse de E. abyssinica et $100 \mathrm{~kg} / \mathrm{ha}$ des cendres (Mukendi et al., 2017, Yalombe et al., 2017). Le semis a été effectué le 17/11/2017 aux écartements de $60 \mathrm{~cm}$ dans la ligne et $50 \mathrm{~cm}$ entre les lignes en raison d'un tubercule par emplacement, soit sept jours après enfouissement de ces engrais organiques. Les observations ont porté sur les paramètres de croissance et de rendement de la culture. Les 
variables observées à la phase végétative sont le nombre de feuilles, la hauteur des plants, le nombre de ramification et le diamètre au collet tandis que ceux de rendement ont été observés à la récolte. Ces variables sont le nombre de tubercules, le diamètre du tubercule et le rendement par hectare

\section{Analyse statistique}

Les données collectées ont été soumises à l'analyse de variance (ANOVA) à l'aide du logiciel Munitab 16.0 et les moyennes ont été séparées en utilisant le test de Student au seuil de $5 \%$.

\section{RESULTATS}

Le taux de levée de la pomme de terre varie, en moyenne, entre 44,8 et $72,1 \%$ (Tableau 1). Les résultats obtenus de l'analyse de la variance montrent que les matières organiques apportées n'influencent pas significativement la levée de la pomme de terre $(\mathrm{P}>0,05)$. Sinon, le traitement témoin montre que les parcelles ayant reçus des apports organiques accusent un taux de levé moyen inférieure. Le Tableau 1 montre qu'il n'y a aucune différence significative.

L'allure de la croissance des plants de la pomme de terre 45 jours après semis a pris en considération les variables nombre de feuilles, hauteur des plants, nombre de ramifications et le diamètre au collet. Le Tableau 2 illustre ces résultats.

Il ressort du Tableau 2 que les matières organiques apportées n'ont pas un effet significatif sur le nombre de feuilles, la hauteur des plants, le nombre de ramifications et le diamètre au collet $(\mathrm{P}>0,05)$.

Les variables de production ont été observées et mesurées après la récolte de la pomme de terre. Les résultats relatifs à ces variables sont consignés dans le Tableau 3 . Il ressort de l'analyse de la variance qu'il existe un effet significatif pour le diamètre des tubercules $(\mathrm{P}<0,05)$.

En ce qui concerne le paramètre nombre de tubercules, la pomme de terre amendée à la cendre présente une valeur moyenne légèrement supérieure à celle ayant été amendée à la bouse de vache respectivement 4,33 et 3,33. Par ailleurs, la pomme de terre installée sur le sol enrichi à Entada abyssinica enregistre une valeur moyenne faible $(2,667)$, similaire au témoin ayant enregistré 2,33 tubercules.

Pour ce qui est du diamètre des tubercules, il ressort que le diamètre supérieur est obtenu avec le sol enrichi à la cendre $(9,66$ $\mathrm{mm}$ ). La valeur intermédiaire est remarquée au sol nourris à la matière organique à base de bouse de vache, soit 7,26 mm. Par contre, la plus petite valeur est signalée dans le sol témoin non enrichi aux matières organiques (4 $\mathrm{mm})$.

Ensuite, les résultats obtenus sur le rendement par hectare montrent que le rendement le plus élevé est obtenu dans la parcelle ayant été amendée à la cendre soit 1tonne/ha. Le rendement le plus faible est remarqué au sol ayant reçu la matière organique à base de Entada abyssinica $(0,6667$ tonne/ha) (Tableau 3).

Tableau 1 : le taux de levée de pomme de terre.

\begin{tabular}{ll}
\hline TRAITEMENTS & Taux de levée en \% \\
\hline Témoin & $72,167^{\mathrm{a}} \pm 14,70$ \\
Bouse & $62,933^{\mathrm{a}} \pm 11,55$ \\
Cendre & $61,067^{\mathrm{a}} \pm 9,64$ \\
Entada & $44,867^{\mathrm{a}} \pm 20,04$ \\
\hline C.V $(\%)$ & 23,70 \\
PPDS $(5 \%)$ & N.S \\
\hline CV : coefficient de variation ; PPDS : plus petite différence significative ; N.S : non significatif.
\end{tabular}


Tableau 2 : Allure de croissance de la pomme de terre à Kabinda.

\begin{tabular}{lllll}
\hline Traitements & NBF & HPL & NBR & DC \\
\hline Témoin & $6,7000^{\mathrm{a}} \pm 1,30$ & $3,33^{\mathrm{a}} \pm 1,04$ & $1,733^{\mathrm{a}} \pm 0,83$ & $2,600^{\mathrm{a}} \pm 0,00$ \\
Bouse & $8,3333^{\mathrm{a}} \pm 1,90$ & $4,66^{\mathrm{a}} \pm 1,25$ & $2,466^{\mathrm{a}} \pm 0,23$ & $2,733^{\mathrm{a}} \pm 0,11$ \\
Cendre & $9,6667^{\mathrm{a}} \pm 1,85$ & $4,00^{\mathrm{a}} \pm 1,32$ & $2,666^{\mathrm{a}} \pm 0,46$ & $2,466^{\mathrm{a}} \pm 0,11$ \\
Entada & $8,1000^{\mathrm{a}} \pm 1,05$ & $4,00^{\mathrm{a}} \pm 0,86$ & $2,866^{\mathrm{a}} \pm 0,23$ & $2,666^{\mathrm{a}} \pm 0,41$ \\
\hline C.V $(\%)$ & 21,57 & 32,63 & 23,73 & 7,54 \\
PPDS $(5 \%)$ & N.S & N.S & N.S & N.S
\end{tabular}

CV : coefficient de variation; PPDS : plus petite différence significative. NBF : Nombre des feuilles ; HPL : hauteur des plants ; NBR : nombre de ramifications. DC : diamètre au collet en $\mathrm{cm}$.

Tableau 3 : Paramètres de production de pomme de terre à Kabinda.

\begin{tabular}{llll}
\hline Traitements & $\begin{array}{c}\text { Nombre de } \\
\text { tubercules }\end{array}$ & Diamètre de tubercules en cm & Rendement en tonne/ha \\
\hline Témoin & $2,33^{\mathrm{a}} \pm 0,57$ & $4,00^{\mathrm{b}} \pm 1,13$ & $0,83^{\mathrm{a}} \pm 0,62$ \\
Bouse & $3,33^{\mathrm{a}} \pm 1,52$ & $7,26^{\mathrm{a}} \pm 2,04$ & $0,73^{\mathrm{a}} \pm 0,40$ \\
Cendre & $4,33^{\mathrm{a}} \pm 0,57$ & $9,60^{\mathrm{a}} \pm 1,73$ & $1,00^{\mathrm{a}} \pm 0,50$ \\
Entada & $2,666^{\mathrm{a}} \pm 1,52$ & $5,06^{\mathrm{b}} \pm 1,72$ & $0,66^{\mathrm{a}} \pm 0,28$ \\
\hline C.V $(\%)$ & 35,31 & 29,50 & 59,70 \\
PPDS $(5 \%)$ & N.S & 1,61 & N.S \\
\hline
\end{tabular}

CV : coefficient de variation ; PPDS : plus petite différence significative, N.S : non significatif.

\section{DISCUSSION}

En ce qui concerne le taux de levée, tous les traitements n'ont pas influencé la germination des tubercules de pomme de terre car cette variable est tributaire des potentialités génétiques de la semence (Yalombe et al., 2017). Les résultats obtenus sur la taille des plants montrent que l'allure de croissance des plants témoins est largement inférieure à celle des plantes fertilisées. La différence de croissance des plants de la pomme de terre observée avec les traitements fertilisés peut être liée à la richesse chimique des amendements organiques utilisés (Tableaux 2). Quant au nombre de feuilles, le plus grand nombre de feuilles est obtenu au sol enrichi à la cendre qui se démarque comparativement aux autres traitements durant la phase d'observation. Ces résultats sont en accord avec ceux obtenus par Ognalaga et al. (2016, 2017) et par Herbert et Breton (2008). Ces résultats s'expliqueraient par le fait que la cendre ramène le $\mathrm{pH}$ vers la neutralité, grâce au remplacement des ions $\mathrm{H}^{+}$et $\mathrm{Al}^{++}$sur le complexe absorbant par les ions basiques échangeables. Les autres variables végétatives notamment le diamètre au collet, le nombre de ramifications et la hauteur de la plante ont présenté des moyennes statistiquement non différentes entre elles. L'analyse statistique de la variance n'a pas permis de mettre en évidence les différences entre les amendements, qui spécialement devraient exister entre les types de matières organiques au stade croissance. Cette observation n'est pas en relation parfaite avec celle observée par Mukendi et al. (2017a). Ces derniers ont 
démontré que la matière organique a un effet positif sur les paramètres de croissance de la culture céréalière de maïs. Mais les résultats obtenus sur le diamètre au collet ont montré que la bouse de vache accroit le diamètre au collet de la pomme de terre, la valeur moyenne d'accroissement étant d'ordre de $0,133 \mathrm{~cm}$ par rapport au traitement témoin traduisant la nécessité d'amender les sols carencés en azote et en phosphore. Ces résultats peuvent être justifiés par le fait que la bouse de vache apporterait une amélioration des propriétés physico-chimiques du sol en éléments majeurs tel que décrit par Ognalaga et al. (2017) sur la culture du Manioc. Selon Ognalaga et al. (2015), le phosphore et l'azote sont des éléments fertilisants majeurs qui assurent des bonnes performances pour la croissance et le rendement des végétaux. Mukendi et al. (2017a) soulignent que la croissance d'une culture dépend de la disponibilité des nutriments par les fumures apportées. Plusieurs littératures soutiennent ces résultats. Cette bouse de vache présente un $\mathrm{pH}$ alcalin qui a pu améliorer celui du sol (Mulaji, 2011 ; Ognalaga et al., 2014).

Les effets observés au Tableau 3 se rapportant au diamètre des tubercules, il s'observe des différences significatives au seuil de 5\% de probabilité. La pomme de terre sous l'application de la cendre a donnée des tubercules ayant un diamètre supérieur $(9,60 \pm$ $1,73 \mathrm{~mm}$ ) par rapport aux tubercules de la pomme de terre issue du sol témoin $(4,00 \pm$ $1,13 \mathrm{~mm})$. Ce résultat peut être expliqué par le fait que les cendres permettraient de corriger la structure et l'acidité du sol en fournissant des éléments nutritifs tels que les cations calcium $\left(\mathrm{Ca}^{++}\right)$et le magnésium $\left(\mathrm{Mg}^{++}\right)$aux plantes. Ces matières organiques à base de la cendre produisent les rendements supérieurs $(1,00 \pm$ $0,50 \mathrm{t} / \mathrm{ha}$ ) comparativement aux autres matières organiques à base de la bouse $(0,73 \pm 0,40 \mathrm{t} / \mathrm{ha})$ et à Entada $(0,66 \pm 0,28 \mathrm{t} / \mathrm{ha})$. La richesse des cendres en oligoéléments confirme les résultats obtenus. Ainsi nos observations rejoignent celles de Lickaz (2002) et Herbert et Breton (2008) démontrant que les cendres permettent d'augmenter la productivité des cultures et les revenus agricoles. Selon Ognalaga et al.
(2015), les cendres à base de chromoleana odorat renferment des teneurs importantes en éléments indispensables comme le $\mathrm{Ca}, \mathrm{Mg}, \mathrm{P}$ et $\mathrm{Na}$ pour la nutrition végétale. Ces auteurs expliquent que les matières organiques exercent tous une influence sur le rendement de la pomme de terre, cette influence est attribuee à la richesse en éléments minéraux de ces matières organiques (Sikora et Szmidt, 2001). Les fumures organiques sont riches en Azote. Selon Douglass et al. (2003), l'azote est un élément constitutif de la chlorophylle, il est un facteur déterminant dans la croissance et la détermination du rendement des plantes. Ces fumures organiques modifient les caractéristiques du sol en jouant le rôle de capacité d'échange cationique, capacité de rétention d'eau. Toutefois, les résultats obtenus rejettent ceux obtenus par Porter et Ocaya (2008) montrant que la fumure organique à base de la cendre n'augmentait pas la gale commune de la variété résistante de la pomme de terre. Les comportements qui se dégagent d'autres amendements seraient liés d'une part à la propriété intrinsèque de ladite variété et d'autre part aux conditions climatiques du milieu d'étude. Le rendement obtenu avec le traitement Entada $(0,66 \pm 0,28 \mathrm{t} / \mathrm{ha})$ laisse penser que l'excès en azote a été préjudiciable pour la pomme de terre (Chabi et al., 2012). Bressoud et al. (2003) expliquent l'amendement de sol doit être mesurée et limitée pour éviter l'excès d'azote. Selon Sou et al. (2005), un apport excessif d'azote peut entraîner une diminution de rendement. Ces résultats peuvent recommander la pratique de la cendre à base des inflorescences des palmiers à huile sous diverses applications, tant pour les zones urbaines que périurbaines de la région de la région d'étude.

\section{Conclusion}

L'objectif de cette étude était d'évaluer les effets des amendements organiques sur la croissance et le rendement de la pomme de terre (Solanum tuberosum) dans les conditions agro-écologiques de Kabinda. Les résultats obtenus montrent que les cendres ont influencées la croissance et la production de la culture expérimentale à Kabinda. Les données 
phénologiques rapportent qu'il existe une différence entre les différentes matières organiques. Le plus grand nombre de feuilles est démarqué nettement au traitement cendre $(4,33)$ par rapport au témoin $(2,33)$ durant la phase d'observation. Quant aux paramètres de production, le nombre des tubercules et le rendement par hectare n'ont pas été très distinctif entre les différentes matières organiques tandis que le diamètre des tubercules semble être significatif au seuil de $5 \%$ de probabilité. Les résultats ont en plus montré que la pomme de terre sous l'application de la cendre a donnée des tubercules ayant un diamètre supérieur de 9,6 $\mathrm{cm}$ comparativement aux tubercules de pomme de terre issues du traitement témoin non amendé $6,7 \mathrm{~cm}$. Sur la base des matières organiques évaluées, les cendres peuvent être utilisées par les agriculteurs dans la région de Kabinda vue leur influence positive sur le rendement en tubercule de pomme de terre, mais aussi leur disponibilité ou accessibilité à moindre coût dans la ville de Kabinda.

\section{CONFLIT D'INTERETS}

Les auteurs de cet article déclarent qu'il n'existe aucun conflit d'intérêt en rapport à ce travail.

\section{CONTRIBUTIONS DES AUTEURS}

Les auteurs ont été actifs à la conception de cet article. ANN est l'auteur principal. Il a défini les protocoles expérimentaux ; HMB a fait les analyses statistiques; GKM, AYY et MMM ont apporté un appui technique ; LNN et LLB ont corrigé le manuscrit et dirigé le travail.

\section{REMERCIEMENTS}

Les auteurs adressent leurs sincères remerciements à tous ceux qui ont lu et amélioré la qualité de ce manuscrit.

\section{REFERENCES}

Biaou O, saidou A, Bachabi F, Padonou G et Balogoun I. 2017. Effet de l'apport de différents types d'engrais organiques sur la fertilité du sol et la production de la carotte (Daucus carota L.) sur sol ferralitique au sud Bénin. Int. J. Biol. Chem. Sci., 11(5): 2315-2326. DOI : https://dx.doi.org/10.4314/ijbcs.v11i5.29

Douglas JT, Aitken MN, Smith CA. 2003. Effects of five non-agricultural organic wastes on soil composition and on the yield and nitrogen recovery on Italian ryegrass. Soil Use Man., 19: 135-138.

FAO. 2016. Nature et Faune: Améliorer la gestion des ressources naturelles pour la sécurité alimentaire en Afrique, 30p.

FAO. 2012. L'état de l'insécurité alimentaire dans le monde, la croissance économique est nécessaire mais elle n'est pas suffisante pour accélérer la réduction de la faim et de la malnutrition, 73p.

Herbert H, Breton B. 2008. Recyclage agricole des cendres de bois au Québec-Etat de la situation, impact et bonnes pratiques agro-environnementales. Agrosolutions, 12(2): 18-33.

Honfoga. 2007. Vers des systèmes privés efficaces d'approvionnement et de distribution d'engrais pour une intensification agricole durable au Bénin. University of Groningen, 40p.

IFDC (Centre International pour la Fertilité des Sols et le Développement Agricole). 2002. L'État du Marché des Intrants Agricoles au Bénin. Projet, MIR., p.106.

Lickaz. 2002. Wood ash. An alternative liming material of agricultural soil. Agri-Facts, Pratical information of Albert'a agricultural industry. AGDEX 534-2, Alberta agricultural and Rural development, $6 \mathrm{p}$.

Ministère du Plan. 2005. Monographie du Kasaï Oriental. République Démocratique du Congo, 149p.

Mukendi TR, Mutamba NB, Kabongo MD, Tshilumba MT, Mpoyi BM Et Munyuli MT. 2017a. Évaluation variétale de quelques génotypes de niébé (Vigna unguiculata (L.) Walp) en conditions agro-écologiques de Kabinda, province de Lomami, République Démocratique du Congo. Afrique SCIENCE, 13(2) : 2431. http://www.afriquescience.info

Mukendi TR, Tshilumba MT, Mpoyi BM, Mutamba NB, Kabongo MD, Ilunga TM, 
Ngoie KJ, Ngoyi ND, Munyuli MT. 2017b. Évaluation de la productivité du maiis (Zea mays L.) sous amendements organique et minéral dans la province de Lomami, République Démocratique du Congo. Journal of Applied Biosciences, 109: 10571-10579. DOI : http://dx.doi.org/10.4314/jab.v109i1.2

Mulaji K. 2011. Utilisation des composts de biodéchets ménagers pour l'amélioration de la fertilité des sols acides de la province de Kinshasa (République Démocratique du Congo). Thèse Université de Liège, Belgique, 191p.

Muyayabantu GM, Kadiata BD, Nkongolo KK. 2012. Reponse of maize to different organic and inorganic fertilization regimes in monocrop and intercrop systems in a sub-Saharan Africa region. Journal of Soil Science and Environmental Management, 3(2) : 4248. DOI : 10.5897/JSSEM 11.079.

Ognalaga M, Daglih MM, Samson Daudet MM, Paul OO. 2017. Effet de la bouse de vaches, du NPK 151515 et de l'urée à $46 \%$ sur la croissance et la production du manioc (Manihot esculenta Crantz var 0018) au Sud-Est du Gabon (Franceville). Journal of Animal \& Plant Sciences, 31(3): 5063-5073. DOI : http://www.m.elewa.org/JAPS

Ognalaga M, Moupéla C, Mourendé GA. Et Oyanadigui Odjogui PI. 2016. Effets comparés des cendres de Chromolaena odorata (L.) King R.M. \& H.E. Rob et d'un engrais minéral soluble dans l'eau (NPK 1515 15) sur la croissance et le rendement de l'oseille de Guinée (Hibiscus sabdariffa L.). Tropicultura, 34(3) : 242-252.

Ognalaga M, Odjogui P, Lekambou J, Poligui R. 2015. Effet des écumes à cannes à sucre, de la poudre et du compost à base de Chromolaena odorata (L.) King R.M. \& H.E. Rob sur la croissance de l'oseille de Guinée (Hibiscus sabdariffa L.). Int. J. Biol. Chem. Sci., 9(5) : 2507-2519. DOI : http://dx.doi.org/10.4314/ijbcs.v9i5.22.

Ognalaga M, Itsoma E. 2014. Effet de Chromolaena odorata et de Leucaena leucocephalae sur la croissance et la production de l'oseille de Guinée (Hibiscus sabdariffa L.). Agronomie Africaine, 26 :1-88.

Orwa C, Mutua A, Kindt R, Jamnadass R, Simons A. 2009. Agroforestree Database: a tree reference and selection guideversion 4.0 (http://www.worldagroforestry.org/af/tre $\mathrm{edb} /$ ).

Porter, Ocaya. 2008. Wood ash: Aliming and Nutrient sour for Potato production systems. University of Maine. Hpp//www.neivea.org/Residuals 2008/08 Res-session2-Porter-Wood\%20AshNov08.pdf

Sikora LJ, Szmidt AK. 2001. Nitrogen sources, mineralization rates, and nitrogen nutrition benefits to plants from composts. In Compost Utilization in Horticultural Cropping Systems, Stoffella PJ, Kahn BA (eds). Lewis Publishers : New York, USA ; 287-305.

Yalombe GN, Yalombe YY, Tshibamba MJ, Odia NJ, Kalambaie BMM. 2017. Evaluation de l'effet combiné du chaulage et du Tithonia diversifolia dans la mobilisation du phosphore sur la culture du niébé dans les conditions édapho-climatiques de Mbuji-Mayi en RDC. Journal of Animal \& Plant Sciences, $\quad 33(3)$ : $\quad 5345-5353$. http://www.m.elewa.org/JAPS. 